\title{
The Role of Stocks and Shocks Concepts in the Debate Over Price Versus Quantity
}

\author{
John E. Parsons · Luca Taschini
}

Accepted: 29 October 2012 / Published online: 25 November 2012

(C) The Author(s) 2012. This article is published with open access at Springerlink.com

\begin{abstract}
Recent literature showed that the choice between a price or quantity control depends, in part, on the dynamic structure of cost uncertainty. Temporary shocks to abatement cost favors the use of a price control, while permanent shocks favor a quantity control. Unfortunately, the importance of this assumption to the optimal choice has not yet received wide attention among economists. We analyze the regulatory sproblem in an alternative setting and reproduce these results. Our contribution is the simplicity of the model and the accessibility of the results, which reinforce the critical role played by the assumed structure of uncertainty.
\end{abstract}

Keywords Cap and trade $\cdot$ Permanent shocks $\cdot$ Tax $\cdot$ Transitory shocks

Part of Parsons' research was supported by the MIT Center for Energy and Environmental Policy Research and the MIT Joint Program on the Science and Policy of Global Change and by a grant from the Doris Duke Charitable Foundation. Part of Taschini's research was supported by the Grantham Research Institute on Climate Change and by the Centre for Climate Change Economics and Policy, which is funded by the UK Economic and Social Research Council (ESRC) and Munich Re. This draft has benefited from helpful comments from Sam Fankhauser, Cameron Hepburn, Jake Jacoby, Gib Metcalf, John Reilly, Mort Webster and Federica Buricco and participants in the MIT Emissions Prediction and Policy Analysis seminar.

J. E. Parsons $(\varangle)$

Sloan School of Management MIT, Massachusetts Institute of Technology, E19-411, 77 Massachusetts Ave, Cambridge, MA 02139, USA

e-mail: jparsons@mit.edu

L. Taschini

The Grantham Research Institute on Climate Change and the Environment, London School of Economics and Political Science, London, UK

e-mail: 1.taschini1@1se.ac.uk 
JEL Classification $\mathrm{H} 23 \cdot \mathrm{Q} 28 \cdot \mathrm{Q} 50 \cdot \mathrm{Q} 58$

\section{Introduction}

Two classic alternatives for regulating pollutants are a cap and trade system or a tax. One is a quantity control and the other a price control. Under idealized circumstances the two are equivalent. If the regulator has full information on the parameters of the economy, then there is a simple duality in the problem: A given quantity control yields a specific price, and vice-versa so that it doesn't matter whether it is the price or the quantity which is fixed directly. However, Weitzman (1974) showed that if the regulator has incomplete information about production costs, this duality no longer holds and the final outcome varies depending upon whether a price control or a quantity control is used. If the expected marginal benefit function is flat relative to the marginal cost function, then a price control is preferred. If the marginal benefit function is steeper, then a quantity control is preferred.

Greenhouse gases are an example of "stock pollutants" for which the accumulation of emissions in the atmosphere determines the environmental damage, as opposed the flow of emissions within any specific window of time. In applying the Weitzman result to the specific problem of greenhouse gas emissions, the early literature emphasized this characteristic. As a stock pollutant, the marginal benefit function for abatement within any single period is relatively flat while the marginal cost function slopes sharply, so a price control maximizes welfare given uncertainty on cost, see, for example, Nordhaus (1994) (Ch. 8, fn. 4), Pizer (1999) and Hoel and Karp (2002). Although the choice ultimately depends upon the empirical parameters of the problem, the stock pollutant nature of greenhouse gases appeared to make the outcome obvious.

Later research on the dynamic problem pointed out that the early literature had made a narrow assumption about the dynamic structure of the uncertainty on cost through time. This later literature solved a more general case, and showed that uncorrelated uncertainty on cost across periods (temporary uncertainty) led to a preference for using a price control in each period, while correlated uncertainty on cost (permanent uncertainty) led to a preference for using a quantity control—see, among others, Newell and Pizer (2003) and Karp and Zhang (2005). Consequently, which control is preferred depends crucially on the degree of correlation in costs across periods, together with the other parameters of the problem.

The original result from the early literature, that a price control is superior for stock pollutants like greenhouse gases, achieved wide currency among economists and policy makers. The later revision, noting the important role of the assumption about the dynamics of cost uncertainty, is less widely appreciated. For example, the Stern Review (2006) reports that the stock pollutant character of the greenhouse gas problem unambiguously argues in favor of a price control.

This paper attempts to address the lag in digesting the later research results by reproducing the results in a slightly different model with some useful features. The model is relatively simple, so that the optimal dynamic policy is easily derived and can be handily simulated. The match between alternative extreme assumptions on the dynamic structure on costs and the preference for either a price or quantity control is stark, so that the underlying relationship between the assumption and the result is highlighted. As always, reproducing a fundamental result in a different context helps clarify the essential relationship between the assumptions and the result. 


\section{A Simple Dynamic Model of Emissions}

We analyze emissions within a finite time horizon. This enables us to use simple backward programming techniques to find the optimal dynamic emissions policy. While this means we do not capture the full complexity of the infinite horizon problem, it turns out to be an acceptable tradeoff since we are able to capture the insights of Newell and Pizer (2003) and Karp and Zhang (2005) within this simpler context. The finite time horizon is divided into an arbitrarily large number of periods indexed by $\{i, i=1,2, \ldots, N\}$. Letting the number of periods grow, and the duration of each period shrink, we can approximate the continuous time solution. As we will see, the form of our solution easily incorporates the duration of each period.

Emissions in each period are denoted $q_{i}$, which is a control variable that can be adjusted without constraint. The economy incurs costs that are declining in the rate of emissions, and also a function of a cost parameter, $\theta_{i}$, that evolves over time. We choose the functional form for the economy's costs as to generate a simple optimization condition in a multi-period problem with discounting:

$$
c_{i}\left(q_{i}, \theta_{i}\right)=e^{\theta_{i}-q_{i}} .
$$

With this form, marginal costs are the negative of the cost,

$$
\frac{\partial c_{i}\left(q_{i}, \theta_{i}\right)}{\partial q_{i}}=-e^{\theta_{i}-q_{i}} .
$$

Higher emissions lower cost. Cutting emissions — abatement—increases cost. Increasing the parameter $\theta_{i}$ shifts cost up while also steepening the cost curve, so that both the cost of abatement and the marginal cost of abatement increases. ${ }^{1}$

We evaluate two contrasting specifications of cost uncertainty. In the first specification, shocks to the cost parameter have only a temporary or transitory impact. A shock affects the cost parameter in that period, but has no impact on the expected cost in any future period. In the second specification, shocks to the cost parameter have a permanent impact. A shock affects the cost parameter in that period, and the expected cost in all future periods is incremented by the same amount, too.

The first specification of the cost parameter $\theta_{i}$ is:

$$
\theta_{i}=\theta_{0}+i v+\sigma \epsilon_{i},
$$

where $\theta_{0}$ is the starting cost parameter, $v$ is the constant expected growth rate in the mean cost parameter, and $\epsilon_{i}$ are independent standard normal random variables, i.e. the shocks to the cost parameter. This defines a process that is white noise around a linearly increasing trend. It is comparable to a mean reverting process with full reversion to the growing mean within the period.

The second specification of the cost parameter $\theta_{i}$ is:

$$
\theta_{i}=\theta_{i-1}+\mu+\sigma \epsilon_{i} .
$$

where $\mu$ is the constant expected growth in the cost parameter. This process is a random walk with trend. It is often said that the random walk has "infinite memory". It is in this sense that we say the shocks have a permanent impact on the cost parameter.

These are two extreme special cases of a more general specification, and correspond to the extreme special cases of perfectly correlated or perfectly uncorrelated uncertainty in the

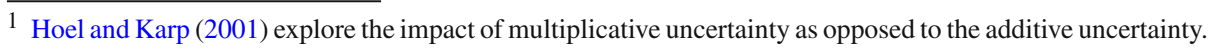


specification used in Newell and Pizer (2003) and Karp and Zhang (2005). A very simple and intuitive presentation of the difference between temporary and permanent uncertainty can be found in an "Appendix" to the on-line working paper version of this paper, Parsons and Taschini (2011). The more general specification is presented in that "Appendix".

The cost-effective emissions policy involves a constant weighing of the updated conditional marginal benefits of a unit of emissions against the updated conditional marginal costs. We focus on a subsidiary problem: What is the dynamic emissions policy that minimizes the cost of regulated agents of achieving a fixed target of aggregate emissions, $\bar{q}$, over the $N$ periods so that:

$$
\sum_{i=1}^{N} q_{i} \leq \bar{q} .
$$

The focus on a simple aggregation of emissions, with emissions in any two periods being perfect substitutes for one another, is a simplified but essential feature of any "stock pollution" problem. The focus on an absolute constraint involves two additional simplifications that require additional discussion. First, defining a fixed, definite constraint abstracts from the problem of weighing the marginal cost of additional abatement in aggregate emissions against the marginal benefit of additional abatement. A fixed, definite constraint would only be optimal if the marginal damage were approximately zero below the constraint and approximately infinite at the constraint. Nevertheless, as we shall see, the solution to the subsidiary cost minimization problem has a very simple structure that successfully captures the key insights of the solutions to the fuller cost-benefit problem analyzed in Newell and Pizer (2003) and Karp and Zhang (2005). Also, in an "Appendix" to the on-line working paper version of this paper, we show that the insights developed here on the characteristics of the cost minimizing dynamic emissions policy carry over when the marginal benefits must be incorporated into the analysis. Second, per Yohe (1978), an absolute constraint like this might yield a bias in favor of a quantity control. Yohe (1978) proposed a model that allows discrepancies between the quantity orders and the actual output achieved by the regulated entities. He notes that, while the relative size of the variance in output under the two opposing modes of control matters, the importance of the comparison still depends on the relative curvature of the cost and benefit functions. While it may be worthwhile to explore a more general version of our model that addresses both of these points, in the interest of preserving the simplicity of our solution, we have restricted ourselves to this less general structure.

We solve for the cost minimizing policy using backward induction. We first set up the general solution format we use, and then we solve for the certainty case and our two extreme uncertainty cases, in which shocks to the cost parameter are either temporary or permanent. A dynamic emissions policy will set emissions in each period conditional on some function of past aggregate emissions and on the current value of the cost parameter. We denote the remaining allowed emissions as we arrive in period $i$ by $\bar{q}_{i}$. Analytically, $\bar{q}_{i+1}=\bar{q}_{i}-q_{i}$ for $i=1, \ldots, N-1$. The choice of emissions will also depend upon the level of the cost parameter, $\theta_{i}$, and so we write emissions as a function of these two parameters, $q_{i}\left(\bar{q}_{i}, \theta_{i}\right)$. We denote the value function in period $i=1, \ldots, N$, as $V_{i}$. Since the target of aggregate emissions is fixed, in the final period when $i=N$, the value function is simply the total cost of remaining emissions to abate:

$$
V_{N}\left(\bar{q}_{N}, q_{N}, \theta_{N}\right) \equiv c\left(q_{N}\left(\bar{q}_{N}, \theta_{N}\right), \theta_{N}\right) .
$$


The cost minimizing emissions level, $q_{N}^{*}\left(\bar{q}_{N}, \theta_{N}\right)$, is the solution of the following problem

$$
\min _{q_{N}} V_{N}\left(\bar{q}_{N}, q_{N}, \theta_{N}\right)
$$

subject to the constraint $\sum_{i=1}^{N} q_{i} \leq \bar{q}$. Given the cost function, the solution is trivial:

$$
q_{N}^{*}\left(\bar{q}_{N}, \theta_{N}\right)=\bar{q}_{N} .
$$

We denote the optimized value function as $V_{N}^{*}$. It is a function of the remaining allowed emissions coming into the period and the realized cost parameter in the period:

$$
\begin{aligned}
V_{N}^{*}\left(\bar{q}_{N}, \theta_{N}\right) & \equiv V_{N}\left(\bar{q}_{N}, q_{N}^{*}\left(\bar{q}_{N}, \theta_{N}\right), \theta_{N}\right) \\
& =c\left(q_{N}^{*}\left(\bar{q}_{N}, \theta_{N}\right), \theta_{N}\right)=c\left(\bar{q}_{N}, \theta_{N}\right) .
\end{aligned}
$$

We will also want to take note of the marginal cost of emissions under this optimal policy which is:

$$
p_{N}^{*}\left(\bar{q}_{N}, \theta_{N}\right) \equiv-\frac{\partial c\left(q_{N}^{*}\left(\bar{q}_{N}, \theta_{N}\right), \theta_{N}\right)}{\partial q_{N}}=c\left(q_{N}^{*}\left(\bar{q}_{N}, \theta_{N}\right), \theta_{N}\right) .
$$

where $p_{N}^{*}\left(\bar{q}_{i}, \theta_{i}\right)$ represents the shadow price and is defined as the negative of marginal cost. For convenience of comparison, we will generally focus on the log of the marginal cost, $\ln \left(p_{i}^{*}\right)=\theta_{i}-q_{i}^{*}$.

In earlier periods, when $1 \leq i<N$, the value function is the total cost of current period emissions plus the discounted expectation of the value function in the subsequent period:

$$
V_{i}\left(\bar{q}_{i}, q_{i}, \theta_{i}\right) \equiv c\left(q_{i}\left(\bar{q}_{i}, \theta_{i}\right), \theta_{i}\right)+\mathbb{E}_{\theta_{i}}\left[V_{i+1}^{*}\left(\bar{q}_{i+1}\left(\bar{q}_{i}, q_{i}\right), \theta_{i+1}\right)\right] .
$$

The uncertainty about $\theta_{i}$ is resolved at the end of each period, therefore the expectation operator is conditioned on the realisation of the cost parameter. The cost minimizing emissions level $q_{i}^{*}\left(\bar{q}_{i}, \theta_{i}\right)$ solves

$$
\min _{q_{i}} V_{i}\left(\bar{q}_{i}, q_{i}, \theta_{i}\right)
$$

The optimized value function is:

$$
V_{i}^{*}\left(\bar{q}_{i}, \theta_{i}\right) \equiv V_{i}\left(\bar{q}_{i}, q_{i}^{*}\left(\bar{q}_{i}, \theta_{i}\right), \theta_{i}\right) .
$$

The marginal cost of emissions is:

$$
p_{i}^{*}\left(\bar{q}_{i}, \theta_{i}\right) \equiv-\frac{\partial c\left(q_{i}^{*}\left(\bar{q}_{i}, \theta_{i}\right), \theta_{i}\right)}{\partial q_{i}}=c\left(q_{i}^{*}\left(\bar{q}_{i}, \theta_{i}\right), \theta_{i}\right) .
$$

The sequence of emissions functions, $q_{i}^{*}\left(\bar{q}_{i}, \theta_{i}\right)$, form the cost minimizing dynamic emissions policy from the perspective of the regulated agent. The sequence of price functions, $p_{i}^{*}\left(\bar{q}_{i}, \theta_{i}\right)$, form the price corresponding to the cost minimizing dynamic emissions policy. ${ }^{2}$

We have not introduced a formal model of asymmetric information on cost between a regulator and firms. In a dynamic context, this would require a specification of the time lag between the evolution of the cost parameter and the evolution of the regulator's updated

2 Our model abstracts from a number of other dynamic factors one might wish to include. For example, Kelly (2005) investigates the general equilibrium effects of price or quantity regulation that are missing in Weitzman and the subsequent literature including this model. Heutel (2012) and Fischer and Springborn (2011) explore dynamic emissions policies in the context of macroeconomic fluctuations in a real business cycle framework. 
information about costs. Moreover, if the regulator is inferring information about the cost parameter from the chosen rate of emissions, then the problem becomes a very complicated dynamic game between the regulator and firms. To finesse this problem, we focus on characterizing features of the cost minimizing dynamic emissions policy, such as the variance in the rate of emissions - the quantity variable — or the variance in the marginal cost of emissionsthe price of emissions, that we believe are robust diagnostics for the viability of regulations focused on quantity or on price. For example, if the cost minimizing dynamic emissions policy yields a quantity that hardly varies in response to shocks to the cost parameter, then it will be relatively easy for a regulator to closely mimic the outcome by means of a quantity control. Conversely, if the cost minimizing dynamic emissions policy yields a marginal cost that varies dramatically in response to shocks to the cost parameter, then it will be relatively hard for a regulator to closely mimic the outcome by means of a price control. The exact measure of success or failure will ultimately depend on the structure of the information flow to the regulator and the game between the regulator and firms. This model does not explore that level of detail.

We now turn to examining the solution to this problem under different circumstances. We begin by solving the certainty case, since this provides useful intuition for the uncertainty cases. We then solve the two contrasting uncertainty cases and show how the degree to which uncertainty is temporary or permanent shapes the cost minimizing emission policy.

\subsection{Certainty Case}

For the certainty case we have $\sigma=0$, so that the dynamics of $\theta_{i}$ reduces to

$$
\theta_{i}=\theta_{0}+i v \text { for } i=1, \ldots, N \text {. }
$$

As shown in the "Appendix", the cost minimizing emissions path has a conveniently simple general form:

$$
q_{i}^{*}=\frac{1}{N-i+1} \bar{q}_{i}-\frac{1}{2}(N-i)(v-r),
$$

and

$$
V_{i}^{*}\left(\bar{q}_{i}, \theta_{i}\right)=i e^{\theta_{i}-q_{i}^{*}}=i e^{\theta_{i}-\frac{1}{N-i+1} \bar{q}_{i}+\frac{1}{2}(N-i)(\nu-r)} .
$$

The log marginal cost of emissions is:

$$
\ln \left(p_{i}^{*}\left(\bar{q}_{i}, \theta_{i}\right)\right)=\theta_{i}-q_{i}^{*}\left(\bar{q}_{i}, \theta_{i}\right)=\theta_{i}-\frac{1}{N-i+1} \bar{q}_{i}+\frac{1}{2}(N-i)(v-r) .
$$

The expressions in Eqs. (3)-(5) are contingent on whatever may be the endowment of remaining allowed emissions coming into the period, $\bar{q}_{i}$, and they are expressed in terms of the remaining number of periods. Therefore, it is not immediately clear from Eq. (3) how the emissions in different periods compare to one another. In the certainty case, we can readily translate back to an equation that describes emissions in different periods as a function of the total allowed emissions, $\bar{q}$, the total number of periods, $N$, the rate of growth in the cost parameter, $v$, and the discount rate, $r$ :

$$
q_{i}^{*}(\bar{q}, N, v, r)=\frac{1}{N} \bar{q}+\left(i-\frac{N}{2}\right)(v-r)
$$



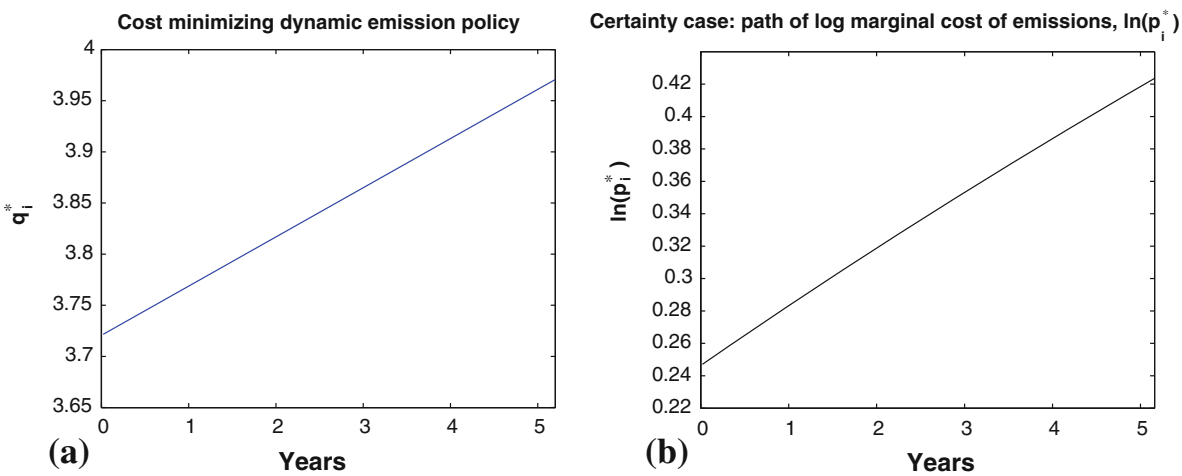

Fig. 1 5-year evolution of the certainty case: cost minimizing dynamic emission policy, $q_{i}^{*}$, (left) and the log of marginal cost of emissions, $\ln \left(\frac{\partial c\left(q_{i}^{*}, \theta_{i}\right)}{\partial q_{i}^{*}}\right)$, (right) for the certainty case. In this example $\bar{q}=1,000, \theta_{0}=5$, $v=0.1, \sigma=0, r=0.05$, and the time step corresponds to a week. a Optimal emission policy $q_{i}^{*}$. $\mathbf{b} \log$ of marginal cost $\ln \left(\frac{\partial c\left(q_{i}^{*}, \theta_{i}\right)}{\partial q_{i}^{*}}\right)$

Transforming the price - the negative of the marginal cost-in log terms for the ease of exposition, $p_{i}^{*}$ can be expressed as:

$$
\ln \left(p_{i}^{*}(\bar{q}, N, v, r)\right)=\theta_{0}-\frac{1}{N} \bar{q}+\frac{N}{2}(v-r)+i r .
$$

To understand the optimal emissions policy in Eq. (3) begin by assuming that $v=r$, so that the cost parameter is growing at a rate equal to the discount rate. In that case, the optimal policy is to allocate to period $i$ a pro rata share of the remaining allowed emissions, $\frac{1}{N-i+1} \bar{q}_{i}$. Application of this policy to successive periods means that emissions are equal in every period, $\frac{\bar{q}}{N}$. The (log) marginal cost of emissions rises, $\theta_{0}-\frac{\bar{q}}{N}+i r$, but at a rate equal to the discount rate, so that the discounted marginal cost is constant across all periods. When $v \neq r$ the optimal policy is to adjust the pro-rata allocation in period $i$ to reflect the differential between the growth rate on the cost parameter and the discount rate. The adjustment assures that emissions increase linearly through time at the rate $v-r$, as seen in Eq. (6) and shown in Fig. 1a, so that the marginal cost of abatement grows at the discount rate, $r$, as seen in Eq. (7) and reported by Fig. 1b. If $v \geq r$, and the underlying cost parameter is growing at a rate greater than the discount rate, then this adjustment leads to reducing the rate of emissions now, in period $i$, increasing the realized marginal cost today, so as to preserve allowed emissions for the later periods when the cost parameter is higher, thus reducing the growth rate in the realized marginal cost to equal the discount rate.

An important feature to take note of in the solution to this certainty case is that the optimal emissions level, $q_{i}^{*}$, is independent of the realized cost parameter, $\theta_{i}$. The cost minimizing emissions path is fully determined by (i) the quantity of emissions being targeted relative to the time remaining, and (ii) the rate of growth in the cost parameter relative to the discount rate. The level of the cost parameter does not enter the equation. If we change the current value of the cost parameter, we don't change the cost minimizing emissions policy! This fact significantly aids our solution of the cost minimizing policy when the evolution of the cost parameter is uncertain, i.e., when we allow $\sigma>0$, whether for the case of temporary or permanent shocks. 


\subsection{Temporary Uncertainty Case}

As shown in the "Appendix", the general form of the optimal dynamic policy is:

$$
q_{i}^{*}=\frac{1}{N-i+1} \bar{q}_{i}-\frac{1}{2}(N-i)(v-r)-A_{i} \sigma^{2}+\frac{N-i}{N-i+1} \sigma \epsilon_{i}
$$

where

$$
A_{i}=\frac{N-i}{N-i+1}\left(A_{i+1}+\frac{1}{2(N-i)^{2}}\right) \text { for } i=1, \ldots, N-1 \text { and } A_{N}=0
$$

The general form of the optimized value function is:

$$
V_{i}^{*}\left(\bar{q}_{i}\right)=i e^{\theta_{i}-q_{i}^{*}}=i e^{\theta_{i}-\frac{1}{N-i+1} \bar{q}_{i}+A_{i} \sigma^{2}+\frac{1}{2}(N-i)(\nu-r)-\frac{N-i}{N-i+1} \sigma \epsilon_{i}} .
$$

The log price is:

$$
\ln \left(p_{i}^{*}\right)=\theta_{0}+i v-\frac{1}{N-i+1} \bar{q}_{i}+A_{i} \sigma^{2}+\frac{1}{2}(N-i)(v-r)-\frac{1}{N-i+1} \sigma \epsilon_{i}
$$

The optimal emissions policy in Eq. (8) is similar to the certainty case in two of the components: the pro rata share of the remaining allowances, $\frac{1}{N-i+1} \bar{q}_{i}$, and the linear growth factor, $\frac{1}{2}(N-i)(v-r)$. In addition, there is a deduction in the current emissions level, $A_{i} \sigma^{2}$, which is tied to the overall volatility of emissions. This is an adjustment to the inter-temporal allocation of emissions necessitated by the increasing uncertainty about future emissions. Finally, there is the component of emissions that fluctuates with the current realization of costs: $\frac{N-i}{N-i+1} \sigma \epsilon_{i}$. If the remaining number of periods is large, then the coefficient is close to 1 , which means that all of the shock in the cost parameter is absorbed in adjustment to the current level of emissions. This adjustment keeps the current level of marginal cost approximately constant. As the remaining number of periods declines, the coefficient on the quantity adjustment falls, so that only a portion of the shock in the cost parameter is absorbed in adjustment to the current level of emissions. This is because of the fixed aggregate emissions constraint. Any adjustment in the current level of emissions must be compensated for with an opposite adjustment in emissions over all of the remaining periods. The coefficient on the quantity adjustment, $\frac{N-i}{N-i+1}$, results in all periods sharing equally in the increased or decreased marginal cost so as to minimize the aggregate cost impact. When there are fewer remaining periods to share the remaining costs, a larger fraction must be absorbed in the current period. Consequently, as the final period approaches, price reflects a larger and larger portion of the shock of the cost parameter.

These points can be formalized by showing the formula for the variance of emissions and of the $\log$ of price. The variance of emissions and log price one period ahead are:

$$
\operatorname{Var}_{i-1}\left(q_{i}^{*}\right)=\frac{N-i}{N-i+1} \sigma, \quad \text { and } \operatorname{Var}_{i-1}\left(\ln \left(p_{i}^{*}\right)\right)=\frac{1}{N-i+1} \sigma .
$$

The variance of the forecasted emissions and log price at any period, $i$, relative to the starting period, $i=0$, are:

$$
\operatorname{Var}_{0}\left(q_{i}^{*}\right)=\sqrt{\sum_{h=1}^{i-1}\left(\frac{1}{N-h+1}\right)^{2}+\left(\frac{N-i}{N-i+1}\right)^{2}} \sigma .
$$

and

$$
\operatorname{Var}_{0}\left(\ln \left(p_{i}^{*}\right)\right)=\sqrt{\sum_{h=1}^{i}\left(\frac{1}{N-h+1}\right)^{2}} \sigma
$$



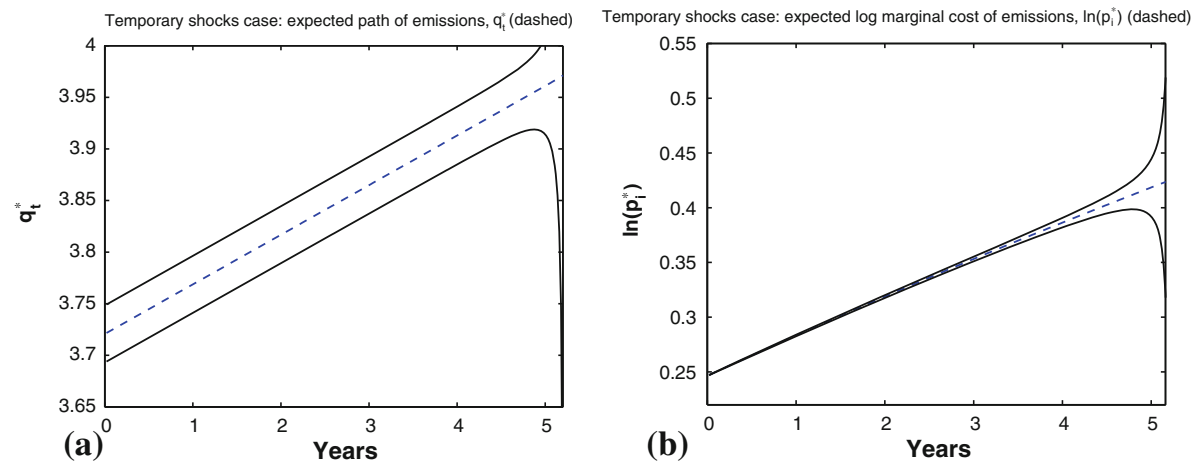

Fig. 2 One-standard deviation confidence bounds around the expected path of the optimal quantity of emissions, $q_{i}^{*}$, (left) and around the expected path of the log of marginal cost, $\ln \left(\frac{\partial c\left(q_{i}^{*}, \theta_{i}\right)}{\partial q_{i}^{*}}\right)$, (right). In this example $\bar{q}=1,000, \theta_{0}=5, v=0.1, \sigma=0.2, r=0.05,5$-year horizon, and the time step corresponds to a week. a Expected path and confidence bounds for $q_{i}^{*}$. b Expected path and confidence bounds for the $\log$ of marginal cost $\ln \left(\frac{\partial c\left(q_{i}^{*}, \theta_{i}\right)}{\partial q_{i}^{*}}\right)$

Figure 2a shows a pair of one-standard deviation confidence bounds around the expected path of the optimal quantity of emissions through time. Figure $2 b$ shows a pair of one-standard deviation confidence bounds around the expected path of the log of marginal cost through time.

\subsection{Permanent Uncertainty Case}

As shown in the "Appendix", the general form of the optimal dynamic policy is:

$$
q_{i}^{*}=\frac{1}{N-i+1} \bar{q}_{i}-\frac{1}{2}(N-i)(\mu-r)
$$

and

$$
V_{i}^{*}\left(\bar{q}_{i}, \theta_{i}\right)=i e^{\theta_{i}-q_{i}^{*}}=i e^{\theta_{i}-\frac{1}{N-i+1} \bar{q}_{i}+\frac{1}{2}(N-i)(\mu-r)} .
$$

The log price is:

$$
\ln \left(p_{i}^{*}\right)=\theta_{i-1}+\mu+\sigma \epsilon_{i}-\frac{1}{N-i+1} \bar{q}_{i}+\frac{1}{2}(N-i)(\mu-r)
$$

With a permanent shock, the cost in all periods is equally higher or lower, so that an adjustment in the current period emissions will not help. In fact, the distribution of emissions over time has no reason to change. The optimal emissions policy in Eq. (9) and represented in Fig. $3 \mathrm{a}$ is identical to the certainty case. Emissions in each period are a proportional fraction of the remaining available allowances as determined by the remaining number of periods over which those allowances must be shared. Emissions are adjusted for an allowance for growth in emissions to match the growth rate in the cost parameter and are independent of the cost parameter itself. Emissions are entirely unresponsive to shocks to the cost parameter. Since none of the cost uncertainty is absorbed by the quantity of emissions, all of the cost uncertainty must be absorbed by the price as shown in Eq. (10). 

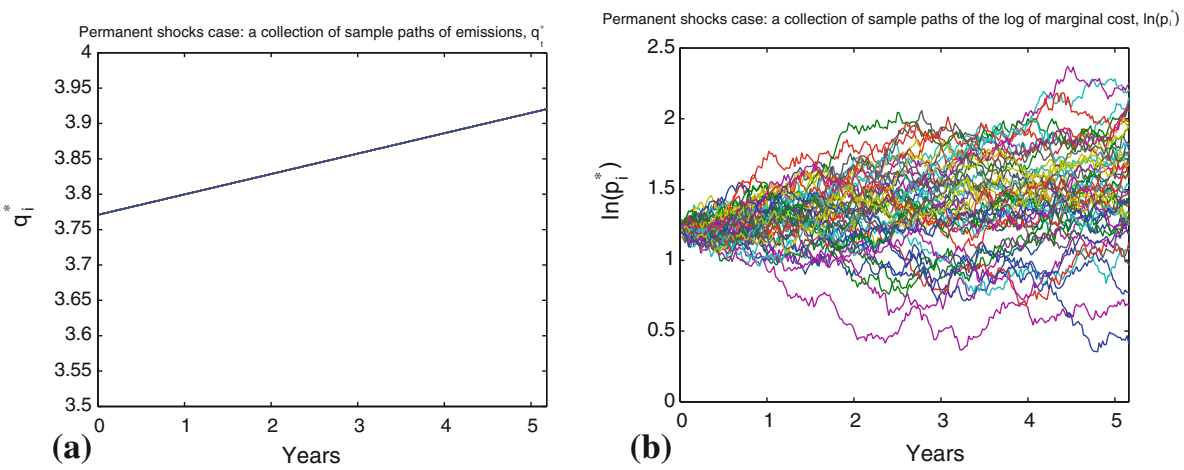

Fig. 3 5-year evolution of the cost minimizing dynamic emission policy, $q_{i}^{*}$, (left) and the log of marginal cost of emissions, $\ln \left(\frac{\partial c\left(q_{i}^{*}, \theta_{i}\right)}{\partial q_{i}^{*}}\right),(r i g h t)$ for the permanent shock case. In this example $\bar{q}=1,000, \theta_{0}=5$, $\mu=0.08, \sigma=0.2, r=0.05$, the time step corresponds to a week, and we consider a set of 50 sample paths. a Optimal emission policy $q_{i}^{*}$. b Collection of sample paths of the log of marginal cost of emissions $\ln \left(\frac{\partial c\left(q_{i}^{*}, \theta_{i}\right)}{\partial q_{i}^{*}}\right)$

These points can be formalized by showing the formula for the variance of emissions and of the $\log$ of price. The variance of emissions and log price one period ahead are:

$$
\operatorname{Var}_{i-1}\left(q_{i}^{*}\right)=0, \text { and } \operatorname{Var}_{i-1}\left(\ln \left(p_{i}^{*}\right)\right)=\sigma .
$$

The variance of the forecasted emissions and log price at any period, $i$, relative to the starting period, $i=0$, are:

$$
\operatorname{Var}_{0}\left(q_{i}^{*}\right)=0, \text { and } \operatorname{Var}_{0}\left(\ln \left(p_{i}^{*}\right)\right)=\sqrt{i} \sigma .
$$

Figure 3a shows the deterministic emissions policy in the face of permanent uncertainty in the cost parameter. Figure $3 \mathrm{~b}$ shows the log of the marginal price.

These two cases provide sharp insight into the different impact that uncertainty in cost should have upon the cost minimizing emissions path depending upon whether it is a temporary uncertainty or a permanent uncertainty. In the case of temporary uncertainty, it is the quantity of emissions that absorbs shocks to the cost parameter, while the price of emissions is relatively constant. In the case of permanent uncertainty, quantity is constant and it is price that absorbs shocks to the cost parameter.

\section{Conclusions}

We constructed a simple model that helps clarify the role of uncertainty in the choice of a price or quantity instrument. Our contribution is the simplicity of the model and the sharpness of the results. In particular, we fully characterize the time path of the optimal quantity of emission responding to shocks to the cost of abatement, whether those shocks temporarily change the cost or permanently change it. If the shocks to cost are exclusively temporary, then it is optimal to adjust the quantity period-by-period, keeping the marginal cost relatively constant. If the shocks are exclusively permanent, then it is optimal to keep the quantity fixed, letting the marginal cost vary as the cost varies. This result provides insight into the optimal instrument choice for a regulator attempting to control emissions by private agents 
with better information on costs, although we do not explicitly model this problem. In the case of temporary uncertainty, when the dynamic emissions policy is to adjust the quantity period-by-period, keeping the marginal cost relatively constant, it is most likely optimal to employ a price instrument. However, in the case of permanent uncertainty, a price instrument will clearly not be optimal since it is the price that ought to absorb all of the shocks to cost. Instead, since quantity should be invariant to the cost shocks period-by-period, a quantity instrument is likely to be optimal. Our results, therefore, reinforce the findings of Newell and Pizer (2003) and Karp and Zhang (2005) that the choice between using a quantity or price instrument hinges on whether or not the uncertainty in costs is correlated across periods.

Our model also provides insight into the operation of a cap-and-trade system that allows banking and borrowing of allowances across periods. The time path of the optimal quantity of emission we derived is also an optimal dynamic allocation of allowances across the periods included under a cap. Therefore, cap-and-trade with banking and borrowing implements the dynamically cost effective emission policy, regardless of the sort of uncertainty. If the capand-trade system faces temporary uncertainty in costs, then it will be the period-by-period quantity of emissions that will fluctuate under the cap-and-trade system, and the price will be relatively constant. If the cap-and-trade system faces permanent uncertainty in costs, then it will be the period-by-period price that will fluctuate under the cap-and trade system, and the quantity of emissions in each period will not be stochastic, but rise deterministically at the rate of growth in costs less the interest rate.

Open Access This article is distributed under the terms of the Creative Commons Attribution License which permits any use, distribution, and reproduction in any medium, provided the original author(s) and the source are credited.

\section{Appendix}

We solve the optimal pollution control problem using recursive substitution. For the sake of exposure, we count periods backwards from the endpoint using the index $j$ to denote periods from the endpoint, with $j=N, \ldots, 2,1$. Recall also that the allowed emissions remaining in the subsequent period is a function of the emissions chosen in the current period, $\bar{q}_{j-1}:=\bar{q}_{j}-q_{j}$. We begin by solving the certainty case, since this provides useful intuition for the uncertainty cases. We then solve the uncertainty case when per period's shock is purely temporary and purely permanent, respectively.

\section{Certainty Case}

When $\sigma=0$, we have the certainty case and the cost parameter follows the dynamics:

$$
\theta_{j-1}=\theta_{j}+v=\theta_{0}+(N-j+1) v
$$

Solving for $j=1$, we have $q_{1}^{*}\left(\overline{q_{1}}, \theta_{1}\right)=\overline{q_{1}}$. Therefore, the value function in the last period $V_{1}^{*}\left(\bar{q}_{1}, \theta_{1}\right)=c\left(\overline{q_{1}}, \theta_{1}\right)=e^{\theta_{1}-q_{1}^{*}}$. For $j=2$, we have

$$
\begin{aligned}
V_{2}\left(\overline{q_{2}}, q_{2}, \theta_{2}\right) & =\mathbb{E}_{\theta_{2}}\left[c\left(q_{2}, \theta_{2}\right)+e^{-r} V_{1}^{*}\left(\overline{q_{1}}\left(\overline{q_{2}}, q_{2}\right), \theta_{1}\right)\right] \\
& =e^{\theta_{2}}\left[e^{-q_{2}}+e^{-(r-v)} e^{-\left(\overline{q_{2}}-q_{2}\right)}\right] .
\end{aligned}
$$


Solving the first order condition for the cost minimizing emissions gives us:

$$
q_{2}^{*}=\frac{1}{2} \overline{q_{2}}-\frac{1}{2}(v-r) .
$$

Substituting this into the value function gives the optimized value function:

$$
V_{2}^{*}\left(\overline{q_{2}}, \theta_{2}\right)=2 e^{\theta_{2}-q_{2}^{*}}=2 e^{\theta_{2}-\frac{1}{2} \overline{q_{2}}+\frac{1}{2}(v-r)} .
$$

For $j=3$, we have

$$
\begin{aligned}
V_{3}\left(\overline{q_{3}}, q_{3}, \theta_{3}\right) & =\mathbb{E}_{\theta_{3}}\left[c\left(q_{3}, \theta_{3}\right)+e^{-r} V_{2}^{*}\left(\overline{q_{2}}\left(\overline{q_{3}}, q_{3}\right), \theta_{2}\right)\right] \\
& =e^{\theta_{3}}\left[e^{-q_{3}}+2 e^{-\frac{\overline{q_{3}}-q_{3}-3 v+3 r}{2}}\right] .
\end{aligned}
$$

Solving the first order condition for the cost minimizing emissions gives us:

$$
q_{3}^{*}=\frac{1}{3} \overline{q_{3}}-\frac{2}{2}(v-r)
$$

Substituting this into the value function gives the optimized value function:

$$
V_{3}^{*}\left(\overline{q_{3}}, \theta_{3}\right)=3 e^{\theta_{3}-q_{3}^{*}}=3 e^{\theta_{3}-\frac{1}{3} \overline{q_{3}}+(\nu-r)} .
$$

For $j=4$, we have

$$
\begin{aligned}
V_{4}\left(\overline{q_{4}}, q_{4}, \theta_{4}\right) & =\mathbb{E}_{\theta_{4}}\left[c\left(q_{4}, \theta_{4}\right)+e^{-r} V_{3}^{*}\left(\overline{q_{3}}\left(\overline{q_{4}}, q_{4}\right), \theta_{3}\right)\right] \\
& =e^{\theta_{4}}\left[e^{-q_{4}}+3 e^{-\frac{\bar{q}_{4}-q_{4}-6 v+6 r}{3}}\right] .
\end{aligned}
$$

Solving the first order condition for the cost minimizing emissions gives us:

$$
q_{4}^{*}=\frac{1}{4} \overline{q_{4}}-\frac{3}{2}(v-r) .
$$

Substituting this into the value function gives the optimized value function:

$$
V_{4}^{*}\left(\overline{q_{4}}, \theta_{4}\right)=4 e^{\theta_{4}-q_{4}^{*}}=4 e^{\theta_{4}-\frac{1}{4} \overline{q_{4}}+\frac{3}{2}(\nu-r)}
$$

Continuing the substitution iteratively, we obtain the general form of the optimal dynamic policy:

$$
q_{j}^{*}=\frac{1}{j} \bar{q}_{j}-\frac{1}{2}(j-1)(v-r) .
$$

and in calendar period $i$, it is:

$$
q_{i}^{*}=\frac{1}{N-i+1} \bar{q}_{i}-\frac{1}{2}(N-i)(v-r) .
$$

The general form of the optimized value function is:

$$
V_{j}^{*}\left(\bar{q}_{j}, \theta_{j}\right)=j e^{\theta_{j}-q_{j}^{*}}=j e^{\theta_{j}-\frac{1}{j} \bar{q}_{j}+\frac{1}{2}(j-1)(v-r)}
$$

and in calendar period $i$, it is:

$$
V_{i}^{*}\left(\bar{q}_{i}, \theta_{i}\right)=i e^{\theta_{i}-q_{i}^{*}}=i e^{\theta_{i}-\frac{1}{N-i+1} \bar{q}_{i}+\frac{1}{2}(N-i)(\nu-r)}
$$


Temporary Shock Case

When the per period's shock is temporary, the cost parameter follows the dynamics:

$$
\theta_{j}=\Theta_{j}+\sigma \epsilon_{j}, \text { where } \Theta_{j} \equiv \Theta_{j+1}+v .
$$

Solving for $j=1$, we have $q_{1}^{*}\left(\bar{q}_{1}, \theta_{1}\right)=\bar{q}_{1}$. Therefore, the value function in the last period $V_{1}^{*}\left(\overline{q_{1}}, \theta_{1}\right)=c\left(\overline{q_{1}}, \theta_{1}\right)=e^{\theta_{1}-q_{1}^{*}}$. For $j=2$, we have

$$
\begin{aligned}
V_{2}\left(\overline{q_{2}}, q_{2}, \theta_{2}\right) & =\mathbb{E}_{\theta_{2}}\left[c\left(q_{2}, \theta_{2}\right)+e^{-r} V_{1}^{*}\left(\overline{q_{1}}\left(\overline{q_{2}}, q_{2}\right), \theta_{1}\right)\right] \\
& =\left[e^{\theta_{2}-q_{2}}+e^{-r} \mathbb{E}_{\theta_{2}}\left[e^{\theta_{1}-\left(\overline{q_{2}}-q_{2}\right)}\right]\right] \\
& =e^{\Theta_{2}}\left[e^{\sigma \epsilon_{2}-q_{2}}+e^{-\left(r-\nu-\frac{\sigma^{2}}{2}\right)} e^{-\left(\overline{q_{2}}-q_{2}\right)}\right]
\end{aligned}
$$

Solving the first order condition for the cost minimizing emissions gives us:

$$
q_{2}^{*}=\frac{1}{2} \overline{q_{2}}-\frac{1}{2}(v-r)-\frac{1}{4} \sigma^{2}+\frac{1}{2} \sigma \epsilon_{2}
$$

Substituting this into the value function gives the optimized value function:

$$
V_{2}^{*}\left(\overline{q_{2}}, \theta_{2}\right)=2 e^{\theta_{2}-q_{2}^{*}}=2 e^{\theta_{2}-\frac{1}{2} \overline{q_{2}}+\frac{1}{2}(\nu-r)+\frac{\sigma^{2}}{4}-\frac{1}{2} \sigma \epsilon_{2}}
$$

For $j=3$, we have

$$
\begin{aligned}
V_{3}\left(\overline{q_{3}}, q_{3}, \theta_{3}\right) & =\mathbb{E}_{\theta_{3}}\left[c\left(q_{3}, \theta_{3}\right)+e^{-r} V_{2}^{*}\left(\overline{q_{2}}\left(\overline{q_{3}}, q_{3}\right), \theta_{2}\right)\right] \\
& =\left[e^{\theta_{3}-q_{3}}+e^{-r} \mathbb{E}_{\theta_{3}}\left[2 e^{\theta_{2}-\frac{\overline{q_{3}-q_{3}-v+r-\sigma^{2} / 2+\sigma \epsilon_{2}}}{2}}\right]\right] \\
& =e^{\Theta_{3}}\left[e^{\sigma \epsilon_{3}-q_{3}}+2 e^{-\frac{\overline{q_{3}-q_{3}-3 v+3 r-3 \sigma^{2} / 4}}{2}}\right]
\end{aligned}
$$

Solving the first order condition for the cost minimizing emissions gives us:

$$
q_{3}^{*}=\frac{1}{3} \overline{q_{3}}-\frac{2}{2}(v-r)-\frac{1}{4} \sigma^{2}+\frac{2}{3} \sigma \epsilon_{3}
$$

Substituting this into the value function gives the optimized value function:

$$
V_{3}^{*}\left(\overline{q_{3}}, \theta_{3}\right)=3 e^{\theta_{3}-q_{3}^{*}}=3 e^{\theta_{3}-\frac{1}{3} \overline{q_{3}}+(\nu-r)+\frac{1}{4} \sigma^{2}-\frac{2}{3} \sigma \epsilon_{3}}
$$

For $j=4$, we have

$$
\begin{aligned}
V_{4}\left(\overline{q_{4}}, q_{4}, \theta_{4}\right) & =\mathbb{E}_{\theta_{4}}\left[c\left(q_{4}, \theta_{4}\right)+e^{-r} V_{3}^{*}\left(\overline{q_{3}}\left(\overline{q_{4}}, q_{4}\right), \theta_{3}\right)\right] \\
& =\left[e^{\theta_{4}-q_{4}}+e^{-r} \mathbb{E}_{\theta_{4}}\left[3 e^{\theta_{3}-\frac{q_{4}-q_{4}-3 v+3 r-3 \sigma^{2} / 4-2 \sigma \epsilon_{3}}{3}}\right]\right] \\
& =e^{\Theta_{4}}\left[e^{\sigma \epsilon_{4}-q_{4}}+3 e^{-\frac{q_{4}-q_{4}-6 v+6 r-11 \sigma^{2} / 12}{3}}\right]
\end{aligned}
$$

Solving the first order condition for the cost minimizing emissions gives us:

$$
q_{4}^{*}=\frac{1}{4} \overline{q_{4}}-\frac{3}{2}(\nu-r)-\frac{11}{48} \sigma^{2}+\frac{3}{4} \sigma \epsilon_{4}
$$


Substituting this into the value function gives the optimized value function:

$$
V_{4}^{*}\left(\overline{q_{4}}, \theta_{4}\right)=4 e^{\theta_{4}-q_{4}^{*}}=4 e^{\theta_{4}-\frac{1}{4} \overline{q_{4}}+\frac{3}{2}(\nu-r)+\frac{11}{48} \sigma^{2}-\frac{3}{4} \sigma \epsilon_{4}}
$$

Continuing the substitution, the general form of the optimal dynamic policy is:

$$
q_{j}^{*}=\frac{1}{j} \bar{q}_{j}-A_{j} \sigma^{2}-\frac{1}{2}(j-1)(v-r)+\frac{j-1}{j} \sigma \epsilon_{j}
$$

where

$$
A_{j}=\frac{j-1}{j}\left(A_{j-1}+\frac{1}{2(j-1)^{2}}\right) \text { for } j=2, \ldots, N \text {, and } A_{1}=0 .
$$

Rewriting in calendar period $i$, we have:

$$
q_{i}^{*}=\frac{1}{N-i+1} \bar{q}_{i}-A_{i} \sigma^{2}-\frac{1}{2}(N-i)(v-r)+\frac{N-i}{N-i+1} \sigma \epsilon_{j}
$$

where

$$
A_{i}=\frac{N-i}{N-i+1}\left(A_{i+1}+\frac{1}{2(N-i)^{2}}\right) \text { for } i=1, \ldots, N-1, \text { and } A_{N}=0 .
$$

The general form of the optimized value function is:

$$
V_{j}^{*}\left(\bar{q}_{j}\right)=j e^{\theta_{j}-q_{j}^{*}}=j e^{\theta_{j}-\frac{1}{j} \bar{q}_{j}+A_{j} \sigma^{2}+\frac{1}{2}(j-1)(\nu-r)-\frac{j-1}{j} \sigma \epsilon_{j}}
$$

and in calendar time:

$$
V_{i}^{*}\left(\bar{q}_{i}\right)=i e^{\theta_{i}-q_{i}^{*}}=i e^{\theta_{i}-\frac{1}{N_{i}+1} \bar{q}_{i}+A_{i} \sigma^{2}+\frac{1}{2}(N-i)(\nu-r)-\frac{N-i}{N-i+1} \sigma \epsilon_{i}} .
$$

\section{Permanent Shock Case}

When the per period's shock is permanent, the cost parameter follows the dynamics

$$
\theta_{i}=\theta_{i-1}+\mu+\sigma \epsilon_{i}
$$

Solving for $j=1$, we have $q_{1}^{*}\left(\overline{q_{1}}, \theta_{1}\right)=\overline{q_{1}}$. Therefore, the value function in the last period $V_{1}^{*}\left(\bar{q}_{1}, \theta_{1}\right)=c\left(\overline{q_{1}}, \theta_{1}\right)=e^{\theta_{1}-q_{1}^{*}}$. For $j=2$, we have

$$
\begin{aligned}
V_{2}\left(\overline{q_{2}}, q_{2}, \theta_{2}\right) & =\mathbb{E}_{\theta_{2}}\left[c\left(q_{2}, \theta_{2}\right)+e^{-r} V_{1}^{*}\left(\overline{q_{1}}\left(\overline{q_{2}}, q_{2}\right), \theta_{1}\right)\right] \\
& =\left[e^{\theta_{2}-q_{2}}+e^{-r} \mathbb{E}_{\theta_{2}}\left[e^{\theta_{1}-\left(\overline{q_{2}}-q_{2}\right)}\right]\right] \\
& =e^{\theta_{2}}\left[e^{-q_{2}}+e^{-\left(r-\mu+\frac{1}{2} \sigma^{2}\right)} e^{-\left(\overline{q_{2}}-q_{2}\right)} e^{\frac{1}{2} \sigma^{2}}\right] .
\end{aligned}
$$

Solving the first order condition for the cost minimizing emissions gives us:

$$
q_{2}^{*}=\frac{1}{2} \overline{q_{2}}-\frac{1}{2}(\mu-r) .
$$

Substituting this into the value function gives the optimized value function:

$$
V_{2}^{*}\left(\overline{q_{2}}, \theta_{2}\right)=2 e^{\theta_{2}-q_{2}^{*}}=2 e^{\theta_{2}-\frac{1}{2} \overline{q_{2}}+\frac{1}{2}(\mu-r)} .
$$


For $j=3$, we have

$$
\begin{aligned}
V_{3}\left(\overline{q_{3}}, q_{3}, \theta_{3}\right) & =\mathbb{E}_{\theta_{3}}\left[c\left(q_{3}, \theta_{3}\right)+e^{-r} V_{2}^{*}\left(\overline{q_{2}}\left(\overline{q_{3}}, q_{3}\right), \theta_{2}\right)\right] \\
& =\left[e^{\theta_{3}-q_{3}}+e^{-r} \mathbb{E}_{\theta_{3}}\left[2 e^{\theta_{2}-\frac{\overline{q_{3}}-q_{3}-\mu+r}{2}}\right]\right] \\
& =e^{\theta_{3}}\left[e^{-q_{3}}+2 e^{-\frac{\bar{q}_{3}-q_{3}-3 \mu+3 r}{2}}\right] .
\end{aligned}
$$

Solving the first order condition for the cost minimizing emissions gives us:

$$
q_{3}^{*}=\frac{1}{3} \overline{q_{3}}-\frac{2}{2}(\mu-r)
$$

Substituting this into the value function gives the optimized value function:

$$
V_{3}^{*}\left(\overline{q_{3}}, \theta_{3}\right)=3 e^{\theta_{3}-q_{3}^{*}}=3 e^{\theta_{3}-\frac{1}{3} \overline{q_{3}}+(\mu-r)} .
$$

For $j=4$, we have

$$
\begin{aligned}
V_{4}\left(\overline{q_{4}}, q_{4}, \theta_{4}\right) & =\mathbb{E}_{\theta_{4}}\left[c\left(q_{4}, \theta_{4}\right)+e^{-r} V_{3}^{*}\left(\overline{q_{3}}\left(\overline{q_{4}}, q_{4}\right), \theta_{3}\right)\right] \\
& =\left[e^{\theta_{4}-q_{4}}+e^{-r} \mathbb{E}_{\theta_{4}}\left[3 e^{\theta_{3}-\frac{\overline{q_{4}}-q_{4}-3 \mu+3 r}{3}}\right]\right] \\
& =e^{\theta_{4}}\left[e^{-q_{4}}+3 e^{-\frac{q_{4}-q_{4}-6 \mu+6 r}{3}}\right] .
\end{aligned}
$$

Solving the first order condition for the cost minimizing emissions gives us:

$$
\begin{aligned}
q_{4}^{*} & =\frac{3}{4} \frac{\overline{q_{4}}-6 \mu+6 r}{3} \\
& =\frac{1}{4} \overline{q_{4}}-\frac{3}{2}(\mu-r) .
\end{aligned}
$$

Substituting this into the value function gives the optimized value function:

$$
V_{4}^{*}\left(\overline{q_{4}}, \theta_{4}\right)=4 e^{\theta_{4}-q_{4}^{*}}=4 e^{\theta_{4}-\frac{1}{4} \overline{q_{4}}+\frac{3}{2}(\mu-r)}
$$

Continuing the substitution, we obtain the general form of the optimal dynamic policy is:

$$
q_{j}^{*}=\frac{1}{j} \bar{q}_{j}-\frac{1}{2}(j-1)(\mu-r) .
$$

and in calendar time $i$, it is:

$$
q_{i}^{*}=\frac{1}{N-i+1} \bar{q}_{i}-\frac{1}{2}(N-i)(\mu-r) .
$$

The general form of the optimized value function is:

$$
V_{j}^{*}\left(\bar{q}_{j}, \theta_{j}\right)=j e^{\theta_{j}-q_{j}^{*}}=j e^{\theta_{j}-\frac{1}{j} \bar{q}_{j}+\frac{1}{2}(j-1)(\mu-r)}
$$

that in calendar time $i$ is:

$$
V_{i}^{*}\left(\bar{q}_{i}, \theta_{i}\right)=i e^{\theta_{i}-q_{i}^{*}}=i e^{\theta_{i}-\frac{1}{N-i+1} \bar{q}_{i}+\frac{1}{2}(N-i)(\mu-r)} .
$$




\section{References}

Fischer C, Springborn M (2011) Emissions targets and the real business cycle: intensity targets versus caps or taxes. J Environ Econ Manag 62:352-366

Heutel G (2012) How should environmental policy respond to business cycles? Optimal policy under persistent productivity shocks. Rev Econ Dyn 15:244-264

Hoel M, Karp L (2001) Taxes and quotas for a stock pollutant with multiplicative uncertainty. J Pub Econ 82:91-114

Hoel M, Karp L (2002) Taxes versus quotas for a stock pollutant. Resour Energy Econ 24:367-384

Karp L, Zhang J (2005) Regulation of stock externalities with correlated abatement costs. Environ Resour Econ 32:273-299

Kelly DL (2005) Price and quantity regulation in general equilibrium. J Econ Theory 125:36-60

Newell RG, Pizer AW (2003) Regulating stock externalities under uncertainty. J Environ Econ Manag 45: 416-432

Nordhaus WD (1994) Managing the global commons. MIT Press, Cambridge

Parsons JE, Taschini L (2011) The role of stocks and shocks concepts in the debate over price vs. quantity. MIT CEEPR working paper 11-002. http://web.mit.edu/ceepr/www/publications/workingpapers/ 2011-002v2.pdf

Pizer WA (1999) The optimal choice of policy in the presence of uncertainty. Resour Energy Econ 21:255-287

Stern N (2006) The economics of climate change. The stern review. Cambridge University Press, Cambridge Weitzman ML (1974) Prices vs quantities. Rev Econ Stud 41(4):683-691

Yohe GW (1978) Towards a general comparison of price controls and quantity controls under uncertainty. Rev Econ Stud 45(2): 229-238 